\title{
Non-Conventional MRI Techniques as an Alternative Role to the Clinical Diagnosis in Alzheimer's Disease
}

\author{
Elisabetta Giugni', Rita Vadalà2, Francesca Romana Pezzella3 ${ }^{3}$, Giuseppe Bomboi ${ }^{1,4}$, \\ Stefano Galletti ${ }^{1}$, Giacomo Luccichenti ${ }^{1,2}$, Carmela Colica ${ }^{1,5}$, Orietta Picconi6, \\ Stefano Bastianello ${ }^{7}$ \\ ${ }^{1}$ European Biomedical Foundation Onlus, Rome, Italy \\ ${ }^{2}$ IRCCS Santa Lucia Foundation, Rome, Italy \\ ${ }^{3}$ Stroke Unit, AOS Camillo-Forlanini Hospital, Rome, Italy \\ ${ }^{4}$ Neurology Unit-II Faculty of Medicine- “Sapienza" University of Rome, Rome, Italy \\ ${ }^{5} \mathrm{CNR}-$ ISN-UOS Pharmacology, Roccelletta di Borgia, Catanzaro, Italy \\ ${ }^{6}$ Opera S.r.I., Genova, Italy \\ ${ }^{7}$ Department of Brain and Behavioral Sciences, University of Pavia, Pavia, Italy \\ Email: rita.vadala@gmail.com
}

Received 13 September 2014; revised 30 October 2014; accepted 15 November 2014

Copyright (C) 2014 by authors and Scientific Research Publishing Inc.

This work is licensed under the Creative Commons Attribution International License (CC BY). http://creativecommons.org/licenses/by/4.0/

(c) (i) Open Access

\begin{abstract}
Improved methods for early diagnosis and non-invasive surrogates for the diagnosis of disease severity in Alzheimer's disease (AD) are becoming the new challenge. Dementia can now be accurately determined through clinical evaluation, cognitive screening, basic laboratory evaluation and structural imaging. Magnetic resonance (MRI) techniques are being evaluated as possible surrogate measures to monitor disease progression. The purpose of this work is to correlate the results of combined advanced MR techniques with neuropsychological performance in order to identify a sensible and sensitive imaging approach to quantify neurodegenerative disease progression. One of the most relevant evidences in our study is the degeneration of the fibers of the corpus callosum in the pathogenesis of cognitive disorders in AD patients, as demonstrated by the relationship between altered neuropsychological tests and reduced FA (Fractional Anisotrophy) values of the corpus callosum in such patients. This data is also integrated by the evidence of anatomic reduction of the total volume of the corpus callosum assessed by FreeSurfer, thus supporting the hypothesis that the "brain disconnects" play a key role in the pathogenesis of AD. Statistical evaluation of regression consisting in the identification of different numerical coefficients that are multiplied by the thickness of the right fusiform value or by the volume of left inferoparietal region and left middle-temporal region, allows us to obtain the predictive numeric value of the related neuropsychological test. Combination of non-conventional magnetic resonance imaging, in-
\end{abstract}


cluding morphometry, spectroscopy, MD (mean diffusivity) and FA evaluation, could be an alternative to clinic in the evaluation of neurodegeneration in AD.

\title{
Keywords
}

\author{
Alzheimer's Disease (AD), Non Conventional MRI Technique, MRI Reproducible Marker
}

\section{Introduction}

In recent years, as a result of the increase in life expectancy, we were witnessing an exponential growth of all chronic and age-related diseases. Among these disorders, dementias are playing an increasing role. Alzheimer's disease (AD), in particular, represents the most common cause of dementia, and it has become a real public health problem with devastating implications for socio-economic impact. AD is typically characterized by impairment of episodic memory in the early stages of the disease, leading to global cognitive impairment with disease progression [1] [2].

Criteria for the clinical diagnosis of Alzheimer's disease (AD) were established by the National Institute of Neurological and Communicative Disorders and Stroke (NINCDS) and the Alzheimer's Disease and Related Disorders Association (ADRDA) workgroup in 1984 [1]. These criteria require that the presence of cognitive impairment and a suspected dementia syndrome be confirmed by neuropsychological testing for a clinical diagnosis of possible or probable $\mathrm{AD}$; however, they need histopathologic confirmation (microscopic examination of brain tissue) for the definitive diagnosis.

These criteria, universally adopted, have been extremely useful, and have survived intactly for more than a quarter of a century. However, with respect to the clinical spectrum of the disease, in the intervening 27 years, important advances have occurred in our understanding of $\mathrm{AD}$, in our ability to detect the pathophysiological process of $\mathrm{AD}$, and in the conceptualization. New criteria for Alzheimer's disease (AD) have recently been developed by an International Working Group in 2007 [2] [3] and the NIA-AA task force in 2011 [4]. These criteria postulate that biomarkers assessment allows the diagnosis of $\mathrm{AD}$ at the predementia stage (as defined through neuropsycological tests) and enhances the accuracy of the differential diagnosis of AD at the dementia stage. Biomarkers assessment includes: Abeta42, tau, and p-tau in the cerebrospinal fluid (CSF), increased cortical uptake of amyloid ligands on PET, posterior cingulate and temporo-parietal hypometabolism on FDG-PET, and medial temporal atrophy on high resolution magnetic resonance (MR). Other biomarkers, useful for the differential diagnosis of AD with Lewy bodies and frontotemporal lobar degeneration, are ioflupane (DaTSCAN) and metaiodobenzylguanidine (123I-MIBG) with SPECT, and serum progranulin [5]. Although criteria should admittedly be used for research, their validation for clinical use would require extensive testing in naturalistic clinical series in multiple memory clinics worldwide. However, several clinical, technical, organizational, and ethical hurdles should be overcome before widespread use in the clinical routine. Despite recent discoveries on the mechanisms and physiopathogenetic, the diagnosis of AD remained a problem of considerable practical importance: currently, in fact, the available diagnostic methods did not allow reaching a definitive diagnosis, with consequent and inevitable problems of choice of treatment, for the neurologist. The role of neuroimaging has been, until now, mostly addressed to rule out other disorders that clinically enter into the differential diagnosis of AD.

The role of diagnostic imaging in the study of dementia is increasingly important because of its increasing role in the field of differential diagnosis through the development of non-conventional MRI techniques, such as morphometric studies [6]-[14], spectroscopy [15]-[19], and functional MRI [20]-[22], able to quantify the brain ultrastructure, metabolism and connectivity.

In the study of dementias, in fact, the techniques of neuroimaging, in addition to their known role in the differential diagnosis, could play an important role in preclinical or early diagnosis and for quantification of disease progression and therapeutic efficacy [23].

As it is known, the differential diagnosis between primary and secondary dementia can be achieved through conventional techniques. However, these neuroimaging techniques, based only on morphological data, do not allow distinguishing the different primitive neurodegenerative diseases, because of poor specificity. The quanti- 
fication of disease progression and therapeutic efficacy is an ambitious goal far from being achieved. MRI is the gold standard system of neuroimaging for the study of the CNS and the dementias.

For the morphological study of the brain, it is possible to distinguish between conventional and unconventional MRI; the latter requires further processing, not commonly performed in clinical routine.

The advantages of non-conventional MRI techniques in the study of AD can be:

1) to assess early signs of neuronal degeneration by observing the early alterations, biochemical and microstructural analysis by metabolic and tensor;

2) to control changes in connectivity of cortical areas;

3) to quantify the cortical and subcortical degeneration, using combined techniques of imaging postprocessing;

4) to evaluate the effect of drug treatments on the parameters of MRI correlation with clinical response and quantification of these effects.

Purpose of this study was to identify valid and effective techniques for the quantification of neuronal degeneration through non-conventional MRI and through aggregate values derived from these same methods which would allow obtaining more sensitive indices in detecting early degeneration with important therapeutic implications.

\section{Materials and Methods}

This study started off in 2010 with the collaboration of the Faculty of Medicine and Psychology, "Sapienza" University of Rome, the European Biomedical Foundation, San Camillo Hospital, Forlanini Foundation, Saint Lucia Foundation and Mondino National Neurological Institute. The supervision of colleagues from the Neuroimaging Section of the University of Szeged, Hungary, was critical in carrying out this study. Protol study was approved by EC of Pavia's IRCCS Mondino National Neurological Institute. The study was divided into several phases. At enrolment, during a neurological examination, patients underwent neuropsychological assessment followed by an MRI examination with both conventional and unconventional sequences. Subsequently, the MRI sequences were post processed and statistical analysis of the data carried out.

All participants signed an informed consent before the study. Each subject was assigned an identification serial number, in accordance with the Privacy Act.

For recruitment in continuous series, all patients reached the observation, after a neurological examination, including neurological history and physical examination. They were recorded progressively and evaluated according to the inclusion/exclusion criteria.

Inclusion criteria were: patients suffering from probable $\mathrm{AD}$, according to the NINDS-ADRDA criteria, aged between 50 and 82 years.

Exclusion Criteria were: the presence of severe neurosensory deficits, schooling under 3 years (in accordance with the minimum schooling of European education system for reading and writing comprehension), the presence of important neuroradiological sign of previous cerebrovascular injury, the presence of serious internal diseases, the lack of compliance that does not ensure complete adherence to the requirements of the protocol for the duration of the study, the presence of history of substance abuse, the presence of factors that contraindicate the execution of an MR exam. We enrolled, in continuing series, 19 patients ( 9 males and 10 females, mean age $74 \pm 8$ years, with a range between 50 and 82 years) with probable $\mathrm{AD}$. An overview of the characteristics of the sample is illustrated in Table 1.

The following procedures were performed during the study:

a) neurological examination

b) neuropsychological assessment included evaluation of mental status and standardized neuropsychological tests in the Italian population. We used MMSE and Milan Overall Dementia Assessment (MODA) to evaluate global cognitive status, in addition we tested other areas such as: selective attention (Attentional matrices, Visual Search and Attention Test-VSAT) sustained attention (TMT trail making test A and B), short-term memory and long-term auditory-verbal (Rey Auditory Verbal Learning Test, Continuous Visual Memory Test), immediate memory (Digit Span Forward and backward test), the logical-deductive ( Raven matrices), language (phonemicverbal fluency and verbal-semantic fluency Test) and praxies (constructional apraxia test). The scores obtained were normalized based on age and education.

c) magnetic resonance examination, performed using an 1.5 Tesla scan (Siemens Healthcare, Erlangen Ger- 
Table 1. Characteristics of the study sample.

\begin{tabular}{cc}
\hline & $\mathrm{Pt}(\mathrm{n}=19)$ \\
\hline AGE & $74+/-8$ \\
SEX (MALE/FEMALE) & $9 / 10$ \\
YEARS OF EDUCATION & $9.5+/-5.6$ \\
MMSE SCORE & $20+/-6$ \\
LENGTH OF DISEASE (MONTH) & $43.1+/-7.7$ \\
\hline
\end{tabular}

many, Magnetom Aera, gradients $33 \mathrm{mT} / \mathrm{m}, 125 \mathrm{~T} / \mathrm{m} / \mathrm{s}$ ) with a standard head coil. The scanning parameters were as follows: FOV: $250 \mathrm{~mm}$; array of acquisition: $256 \times 256$; Thickness of layer: $3 \mathrm{~mm}$; GAP: 0; Acquisition Mode: interlaced; Number of sections: 44 - 48. In order to speed the acquisition, it is used a rectangular FOV with a length of the axis of phase encoding FOV $=3 / 4$. To reduce the acquisition time, the length of the echotrain (fast turbo factor) has been optimized. The tomograms were positioned in the axial plane parallel to the line passing from the upper margin of the anterior commissure and the inferior margin of the posterior commissure (AC-PC line, or bi-commissurale). The acquisition volume has extended beyond the cortex to cover the entire brain. The sections were scanned perpendicular to the sagittal plane defined as the plane passing along the superior sagittal sinus, the falx cerebri, the structures of the median line along the fronto-nasal suture (nasion). The following acquisitions were made: Axial T1-weighted; DP-axial T2 -T2 (triple echo) TE: 13-108-148; TR: 3480; Axial DWI-DTI 12 degree TE 118; TR 7400; Axial 3D magnetization prepared Fast Low Angle Shot (MPRAGE) TE: 2.4 TR: 1730 .

Multivoxel 1H-MRS studies were performed using a standard head neck coil (8NVHEAD NECK). Triplane T2-weighted images were performed to localize the volumes of interest in regions of gray and white matter with macroscopically normal appearance. 1H-MRS spectroscopy was performed with Chemical Shift Imaging (CSI) SE with $\mathrm{TE}=30 \mathrm{~ms}$ with suppression of the water signal in correspondence of the hippocampi and semi-oval centers.

The electronic copy of the MRI examinations was filed with the European Biomedical Foundation, Rome. The computer data of MR images in DICOM format were transferred to a workstation present at the same Foundation. The entire work of post-processing of neuroimaging, was carried out at the European Biomedical Foundation, in collaboration with the Neuroimaging Section of the Neurológiai Clinic of the Albert SzentGyörgyi Medical and Pharmaceutical Center, Faculty of General Medicine of the University of Szeged, as regards the analysis of the images in diffusion. For the entire analytical process, was used the same workstation previously mentioned with a Linux operating system Ubuntu (http://www.ubuntu.com/).

\subsection{Qualitative Analysis of MR Images}

Image analysis was performed independently by experienced operators, unaware of the clinical history resulting from the quantitative analysis. The results were recorded on special forms. They assessed the following characteristics:

1. the "noise" of the image signal, on a three-point scale;

2. motion artifacts, on a three-point scale;

3. other artifacts, which have been described and quantified on a three-point scale;

4. the picture quality, on a five-point scale;

5. These other characteristics of the images were evaluated separately for each MR exam:

6. visibility of T2-hyperintense lesions, on a three-point scale;

\subsection{Post-Processing Analysis of Diffusion MRI}

Diffusion data by 12 degree of freedom affine linear registration were corrected for artefacts to the mean of the non diffusion-weighted reference image [24].

Diffusion tensors at each voxel have been adapted by algorithm included in the FMRIB's Diffusion Toolbox (FDT) of FMRIB's Software Library (FSL v. 4.0, www.fmrib.ox.ac.uk/fsl [25]).

For the whole brain Fractional anisotropy (FA), mean diffusivity (MD), and parallel and perpendicular diffu- 
sivity to the principal diffusion direction were calculated.

Track Based Spatial Statistics (TBSS) method we used in order to reduce the possible errors arising from misalignment of the images [26].

All subjects' FA data were aligned into a common space using the nonlinear registration tool FNIR, in order to obtain a mean FA image [27]-[29].

Each subject's aligned FA data was then projected onto a skeleton map and the resulting data fed into voxel-wise cross-subject statistics.

We also performed a region of interest analysis on the white matter regions whose FA values differed significantly between groups in order to investigate possible differences in FA, MD longitudinal and perpendicular diffusivity.

Connectivity of the identified differences in white matter integrity was defined by probabilistic tractography (FMRIB's Diffusion Toolbox, part of FSL: www.fmrib.ox.ac.uk/fsl/fdt/).

For tractography, we create 5000 streamline samples from each seed voxel to build up a connectivity distribution.

Fitting a multi-fiber model to our diffusion data we had traced pathways through regions of fiber crossing [30].

\subsection{Post-Processing Analysis of Structural MR Images}

The three-dimensional reconstruction of the cortical surface was carried out on the basis of the MPRAGE images using FreeSurfer software [31]. The procedure consisted of the segmentation of the cerebral white matter, in the demarcation of the border between white and gray matter and semi-automatic correction of topological defects resulting from the analysis of the software.

This result was then used as a starting point for analyzing the surface of the areas of white matter, gray and the pial surface with sub-millimeter accuracy. Parcellization of the various regions of interest was also obtained. For each subject, cortical thickness was analyzed with a grid with $1 \mathrm{~mm}$ of space between the two hemispheres. The measures of cortical thickness were mapped on the image obtained from the three-dimensional reconstruction of the brain of each subject. All images were compared with a reference surface using an averaging technique high resolution, based on the surface. The RI obtained from a standard brain has been mapped on the images relative to each participant, using a procedure morphological high spherical size to find the homologous regions in the various subjects. Finally, the volume and the average thickness of the cortical gray matter were analyzed in each region of interest. Cortical thickness was, therefore, rounded in the order of magnitude of $1 \mathrm{~mm}$ to reduce the effect arising from local variations.

For each patient, information about volume, surface area and cortical thickness, with respect to the different anatomic regions, has been obtained

\subsection{Post-Processing Analysis of MRI Spectroscopy}

The investigation of MR spectroscopy was conducted for the temporal, parietal and frontal lobes. The metabolites taken into consideration were the N-acetylaspartate (NAA), choline (Cho) and creatine (Cr).

The volume of interest of the multivoxel section was 10 (anterior to posterior) $\times 8$ (left to right) $\times 2 \mathrm{~cm}$ (length) and it was performed in two different anatomical regions: the first, most caudal, including temporal and occipital lobe, the second, most cranial, including frontal and parietal regions. For acquiring the multivoxel spectroscopy data H-MVS point resolved spectroscopy pulse sequence $(\mathrm{TE}=108 \mathrm{~ms}$; TR $=3480 \mathrm{~ms}$; phase $\times$ frequency, $18 \times 18$ ) was used. A Siemens dedicated software elaborate automatically a post processing phase using standards corrections.

Metabolite signal intensity in NAA, tCr, Cho, and MI was quantified and subsequently, metabolite ratios (NAA/Cr, Cho/Cr, MI/Cr, and NAA/MI), relative to $\mathrm{Cr}$ and MI, was calculated.

\subsection{Post-Processing Analysis of MRI Spectroscopy}

The investigation of MR spectroscopy was conducted for the temporal, parietal and frontal lobes. The metabolites taken into consideration were the $\mathrm{N}$-acetylaspartate (NAA), choline (Cho) and creatine (Cr).

The volume of interest of the multivoxel section was 10 (anterior to posterior) $\times 8$ (left to right) $\times 2 \mathrm{~cm}$ 
(length) and it was performed in two different anatomical regions, the first, most caudal, including temporal and occipital lobe, the second, most cranial, including frontal and parietal regions. H-MVS point resolved spectroscopy (2D PROBE-CSI PRESS) pulse sequence (TE $=108 \mathrm{~ms}$; TR $=3480 \mathrm{~ms}$; phase $\times$ frequency, $18 \times 18$ ) was used for acquiring the multivoxel spectroscopy data. Water suppression $(\geq 98 \%)$ and shimming (linewidth, $<10$ ) were automatically achieved using a variable pulse power and optimized relaxation delay scheme. Post-processing, including phase and frequency correction was performed using Siemens dedicated software.

Metabolite signal intensity in NAA (2.02 ppm), tCr (3.02 and $3.96 \mathrm{ppm})$, Cho (3.22 ppm), and MI (3.56 ppm) was quantified and subsequently, metabolite ratios (NAA/Cr, Cho/Cr, $\mathrm{MI} / \mathrm{Cr}$, and $\mathrm{NAA} / \mathrm{MI}$ ), relative to $\mathrm{Cr}$ and MI, can be calculated.

\subsection{Statistical Analysis}

The DTI data had already partially undergone the process of statistical analysis in the course of their analysis, thanks to the TBSS method. The clinical, neuropsychological and neuroimaging post-processing analysis were subjected to statistical analysis using SPSS 15.0 for Windows, version 15.0.1 (SPSS Inc. 2006). Linear regression was used to estimate the coefficients of the various parameters considered in the determination of the value of cognitive tests (if the coefficient is positive, the relationship is direct; if it is negative, the relationship is inverse). Therefore, a correlation analysis was carried out by calculating the Spearman correlation coefficients (rho). In this study, we calculated the values of rho to assess the relationship between: size of each neuropsychological scale, demographic characteristics of patients; the parameters obtained from the investigation of non-conventional MRI. Each correlation was assessed for statistical significance at $\mathrm{P}<0.05$. When the value of rho is between 0 and +1.00 it means that a variable increases with the increase of the other; when rho is between 0 and -1.00 it means that a variable increases with the decrease of the other. If rho $=0: 00$ to $0: 40$, the correlation between the variables is poor, if rho $=0.40-0.60$ it is fair, if rho $=0.60-0.80$ it is good, if rho $=0.80-1.00$ it is strong.

\section{Results}

Statistical analysis showed a selective correlation between FA values measured in the corpus callosum and the scores at the MMSE, TMT-B, phonemic fluency and RAVLT (delayed mode) and a correlation between FA values of fronto-parietal-temporal regions and the right RAVLT (delayed mode). It was possible to directly visualize the statistical results with the graphical interface fslview, as shown in Figures 1-4.

We achieved these results for the MMSE (Figure 1); for the TMT-B (Figure 2); for the phonemic fluency test (Figure 3); and, therefore, we observed these results for the RAVLT (Figure 4).

We did not get any statistically significant correlation between the values of MD (mean diffusivity) and scores for all neuropsychological tests. Finally, we performed a correlation statistical analysis of the data obtained from the evaluation of the thickness and regional brain volumes, using FreeSurfer, and the neuropsychological tests scores obtained by the subjects with $\mathrm{AD}$.

We observed significant correlations between the various RAVLT score (immediate mode and delayed), and Raven Progressive Matrices test and thickness of different left cortical areas. However, the most significant result was the direct correlation between the score obtained in the MMSE score and the thickness of the right entorhinal cortex, the thickness of the fusiform and the thickness of the inferior-superior temporal pole $(\mathrm{P}>0.01)$ as shown in Figure 5.

We obtained the correlation between the ratio of NAA/Cr and $\mathrm{Cho/Cr}$ measured in the temporal, parietal and frontal lobes, and scores to neuropsychological tests from patients with AD. It emerged, in particular, that there is a high and significant correlation between some neuropsychological tests and the ratio NAA/Cr in the frontal white matter of the right and left frontal gray matter. Moreover, the same analysis showed a significant correlation between the neuropsychological tests and the $\mathrm{Cho} / \mathrm{Cr}$ ratio of the mesial occipital gray matter.

A significant result was finally achieved using multi-modal image analysis: in this way, we have, in fact, observeda relationship between the combined value of the FA of the corpus callosum, its volume and MMSE score. Finally, we performed a statistical analysis of regression between the value of cortical thickness and regional brain volumes and neuropsychological tests.

With this statistical evaluation we have identified different numerical coefficients that, multiplied by the thickness value or by the volume value of the cerebral regional, for which there has been a finding of statistical 


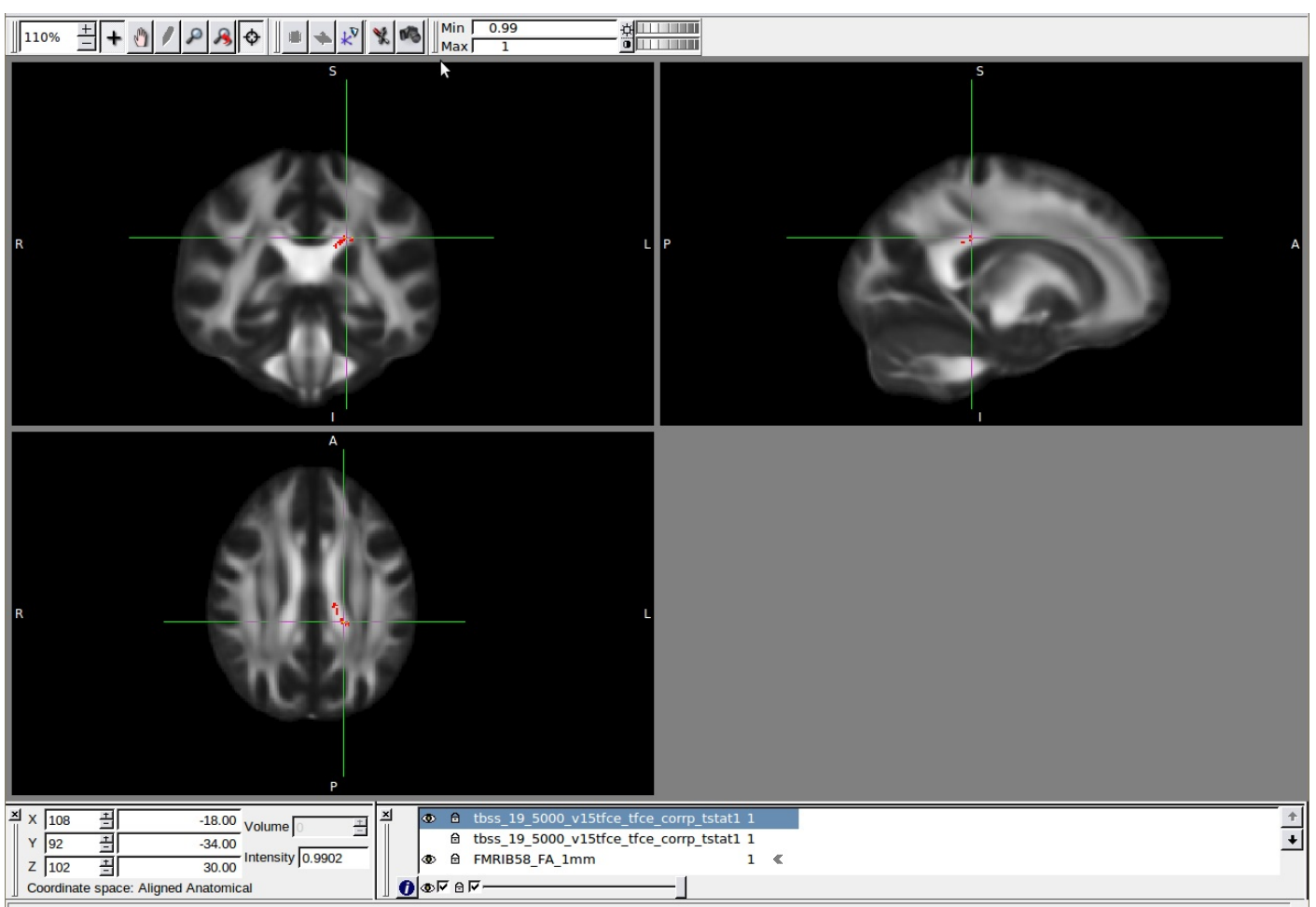

Figure 1. Correlation using TBSS (tracted based spatial statistic) between the score of the MMSE and FA data. The TBSS indicates a reduction of the values of the fractional anisotropy in the left postero-parietal segment of the corpus callosum in patients with AD. The average values of FA are shown in red.

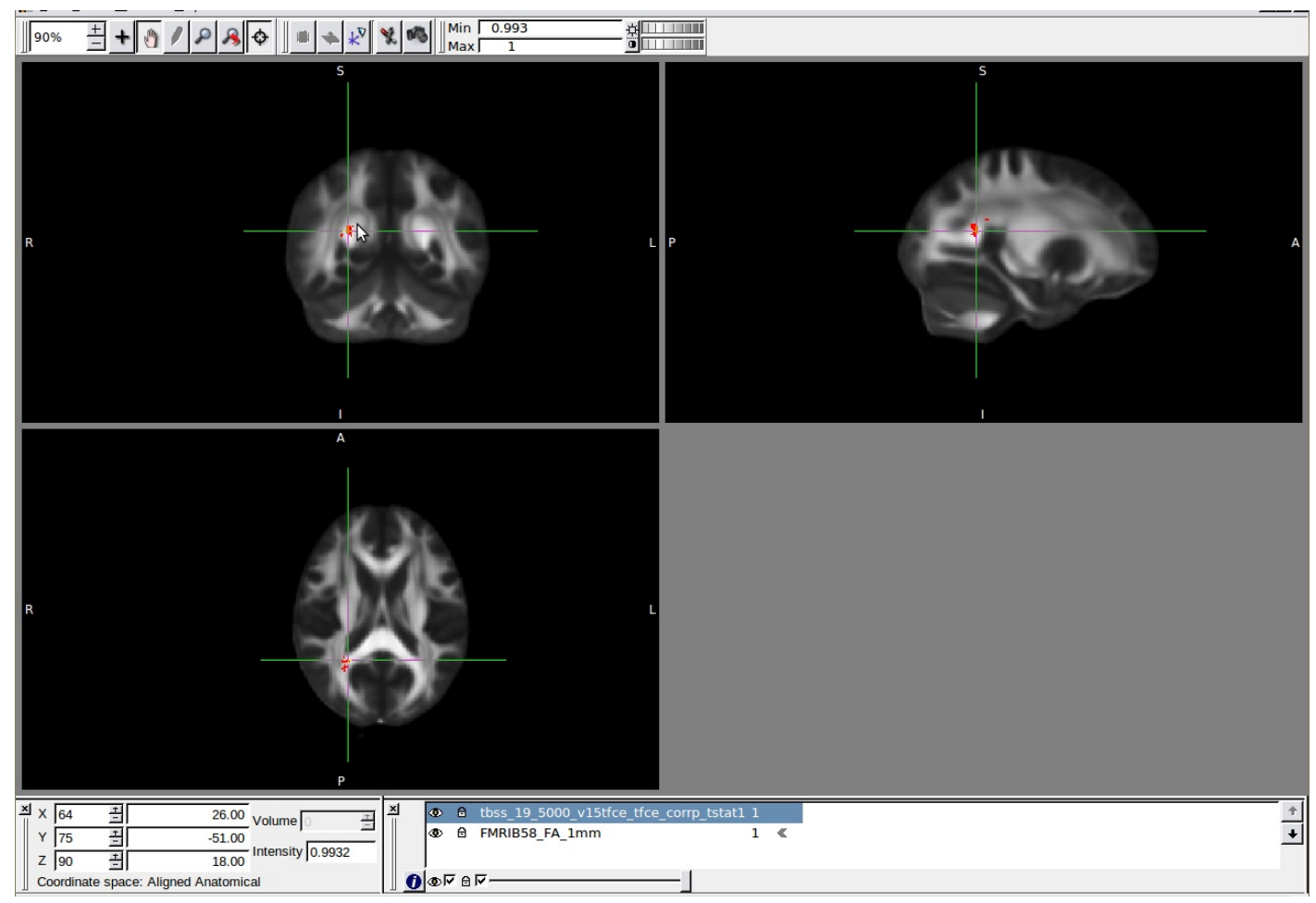

Figure 2. Correlation using TBSS (tracted based spatial statistic) between the score of the TMT-B and FA data. The TBSS indicates a reduction of the values of the fractional anisotropy in the right left postero-parietal segment of the corpus callosum in patients with AD. The average values of FA are shown in red. 


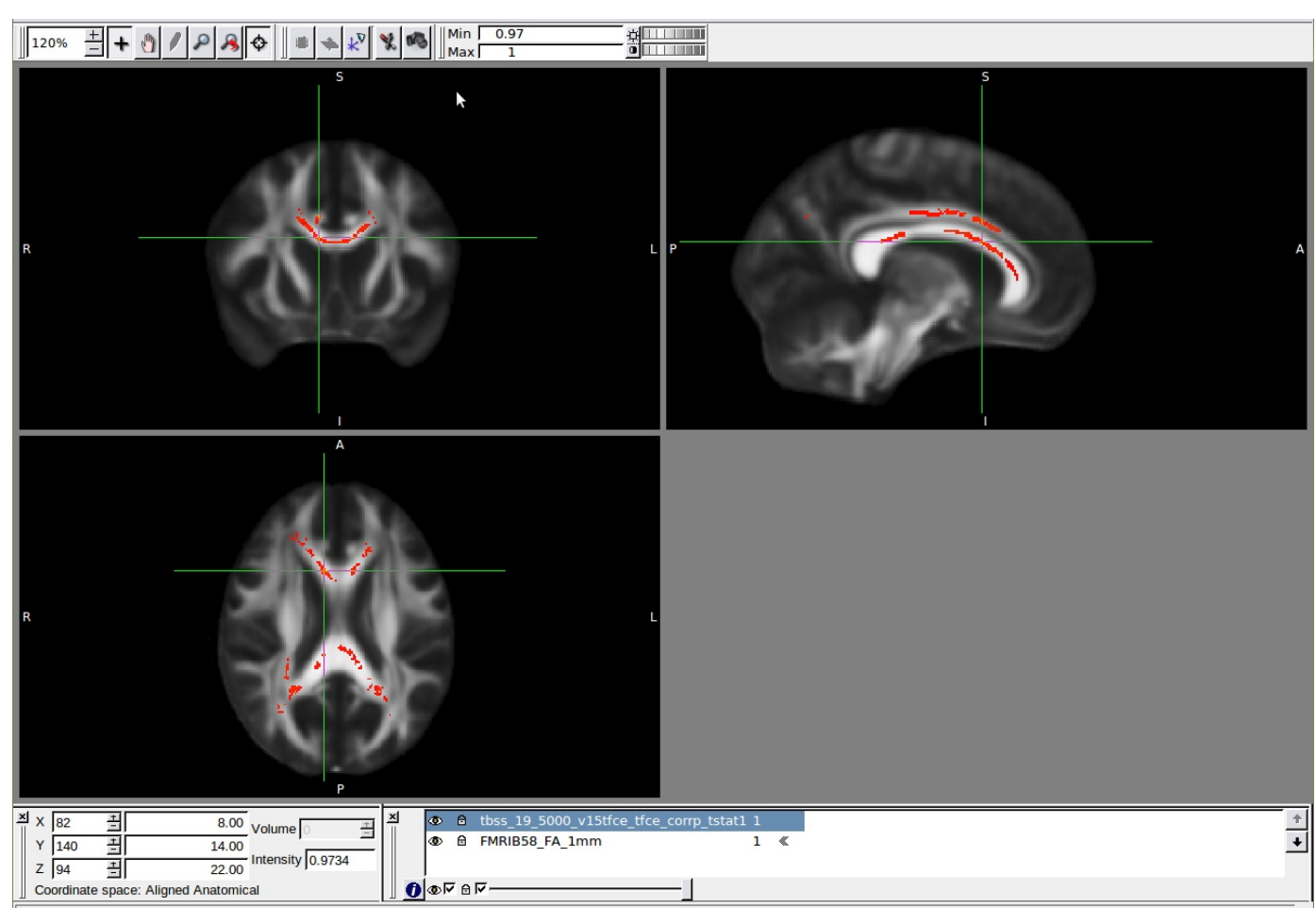

Figure 3. Correlation using TBSS (tracted based spatial statistic) between the score of the FLFON and FA data. The TBSS indicates a reduction of the values of the fractional anisotropy in all the corpus callosum segmentes in patients with $\mathrm{AD}$. The average values of FA are shown in red.

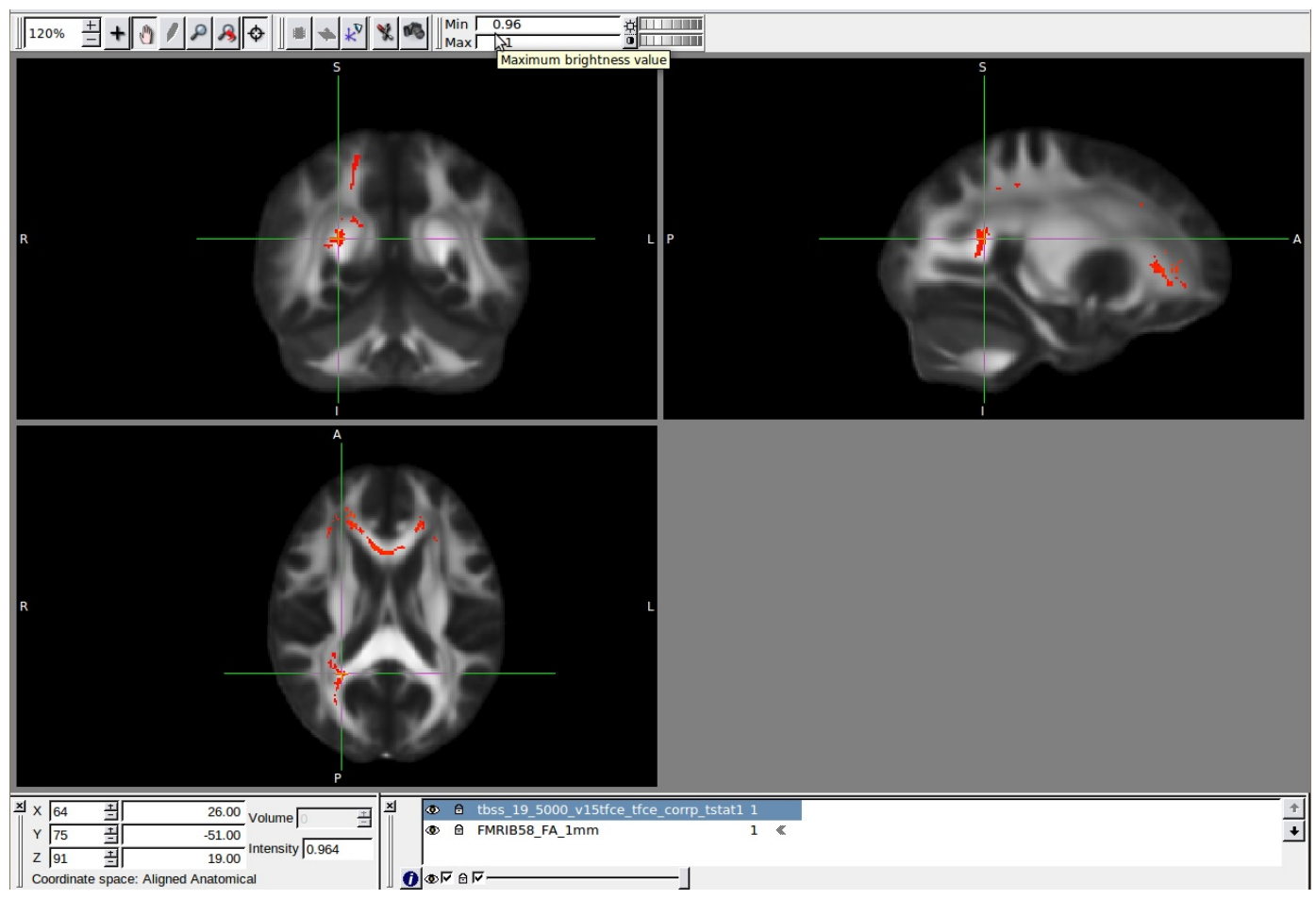

Figure 4. Correlation using TBSS (tracted based spatial statistic) between the score of the RAVLT and FA data. The TBSS indicates a reduction of the values of the fractional anisotropy in the bilateral antero frontal segment and right postero frontal segmento of the corpus callosum in patients with AD. The average values of FA are shown in red. 


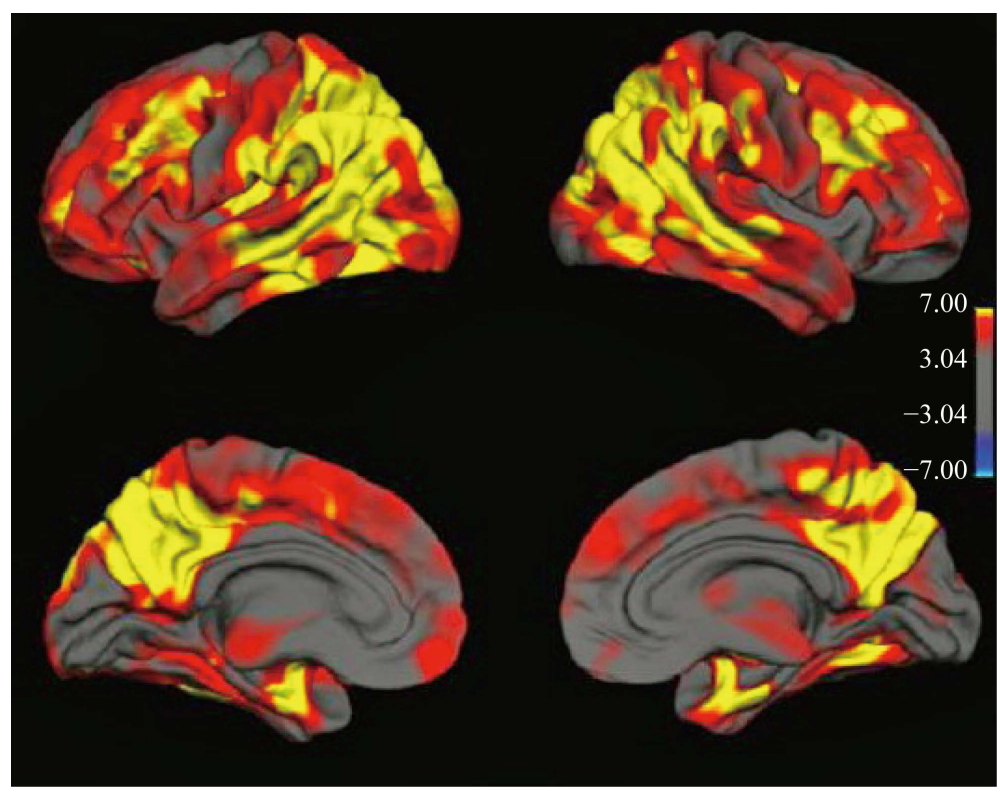

Figure 5. MMSE seemed directly correlated with the thickness of the right entorhinal cortex, with the fusiform area, and with the inferior-superior temporal pole ( $\mathrm{P}>0.01$ ). The color code for the values of $\mathrm{P}$ is expressed by a logarithmic scale from 1 to 5 . Warmer colors represent the regions in which the severity of cognitive impairment correlate more strongly with the reduction of cortical thickness.

significance, allow us to obtain the predictive numeric value of the related neuropsychological test. In particular, we identified a numerical coefficient predictor of MMSE, MODA, REY DIF and FL.FON in the evaluation of regression that correlates with cortical thickness of the right fusiform; a numerical coefficient predictor of REY DIFF that correlates with the volume of left inferoparietal region, and a numerical coefficient predictor FL.SEM that correlates with the volume of left middle-temporal region.

\section{Discussion}

One of the most relevant evidence in our study was that the microstructure of white matter valued TBSS appear to be significantly altered in patients suffering from probable AD.

In particular, the most relevant is the degeneration of the fibers of the corpus callosum in the pathogenesis of cognitive disorders of patients with $\mathrm{AD}$, as demonstrated by the relationship between neuropsychological tests altered and reduced FA values of the corpus callosum of patients with AD. This data is also integrated by the evidence of anatomic reduction of the total volume of the corpus callosum assessed by FreeSurfer.

This "damage" is intimately related mainly to memory interference, verbal and attentional disorders, present in $\mathrm{AD}$. The corpus callosum is the most extensive way of connecting white matter fibers in the brain; the knowledge of its age-related changes and disease is one of the key aspects in order to understand its role in cognitive processes.

Corpus callosum is commonly divided into seven distinct segments, based on the target cortical areas: the orbitofrontal (OF), anterior-frontal (AF), superior-frontal (SF), superior-parietal (SP), posterior parietal (PP), temporal (Temp) and occipital (Occ). The segmentation of the corpus callosum into seven portions, instead of the classic pathologic division into four portions (rostrum, genius, body and splenium) allows a more detailed localization of alterations due to pathological processes.

Based on these findings, we have shown a correlation between the global cognitive performance and global degeneration of the posterior parietal segment of the corpus callosum and a correlation between the overall degeneration of this structure and phonemic functions. From a pathophysiological point of view, these results confirm that different patterns of alteration of the white matter of the corpus callosum are associated with AD. This supports the hypothesis that the "brain disconnects" play a key role in the pathogenesis of $\mathrm{AD}$, contributing to 
cognitive and memory attentional especially in the initial phase of moderate-AD. The ultrastructural data obtained with the data of diffusion MRI should be added to the anatomical correlation between the volume of the corpus callosum and the score of the MMSE. The most significant data is the correlation between the MMSE and the thickness of the entorhinal cortex, the fusiform gyrus and the temporal pole. The figure shows, in fact, on the one hand, the importance of the role of the MMSE as a screening test of global cognitive impairment, given its significant correlation with the atrophy of the structures primarily affected by the AD and, on the other hand, the value of diagnostic assessment of cortical thickness in MA, precisely because of its correlation with the MMSE score.

In addition to these results for the MMSE, the study showed that the cognitive functions which correlate with the atrophic process appear to be memory and logical-deductive.

To the anatomical damage of the cortical temporo-mesial area, is added the evidence of harm to the metabolic nature, identified by our spectroscopic studies. The ratio NAA/Cr in the right frontal white matter correlates with tests indicating disorders of memory and language and also with the MMSE, index of global cognitive decline.

The same ratio, appeared in the left frontal cortex, is correlated with memory disorders and with global cognitive decline, while the Cho/Cr ratio in the right mesial occipital substance correlated with attentional disorders. As it is known, in $\mathrm{AD}$ there is a characteristic and significant decrease of NAA and its parietooccipitale ratio NAA/Cr ratio in the cortex of the left occipital lobe has a sensitivity of $78 \%$ and a specificity of $69 \%$, in predicting the development of dementia in subjects suffering from MCI.

Through the regression statistical analysis, structural and functional imaging techniques have been related to neuropsychological tests. Through this statistical evaluation we have identified different numerical coefficients that, multiplied by the thickness value or by the volume value of the cerebral regional for which there has been a finding of statistical significance, allow us to obtain the predictive numeric value of the related neuropsychological test. In particular, we identified a numerical coefficient predictor of MMSE, MODA, REY DIF and FL. FON in the evaluation of regression that correlates with cortical thickness of the right fusiform; a numerical coefficient predictor of REY DIFF that correlates with the volume of left inferoparietal region, and a numerical coefficient predictor FL. SEM that correlates with the volume of left middle-temporal region.

This data allow us to hypothesize a neuropsicological test surrogate role of magnetic resonance imaging in diagnoses and evaluation of disease progression.

It should be added that the measurements obtained from the data of MR are in line with what has been shown also by neuropathological studies in the differential diagnosis between healthy subjects and patients with AD and they contribute substantially to the improvement of diagnosis, compared with the use of just neuropsychological tests.

\section{Conclusions}

We can therefore conclude that, by analyzing diffusion data together with structural data, our study has highlighted a correlation between in vivo anatomical and ultrastructural parameters and cognitive parameters.

The combination of different methods of evaluating non-conventional MRI morphometry, spectroscopy, MD and FA provides a useful new approach for assessing the anatomical, metabolic and ultrastructural neurodegenerative processes underlying the $\mathrm{AD}$. Unconventional methods of MRI are now widely available on scanners operating at field strength of $1.5 \mathrm{~T}$; to standardize a protocol with predictive parameters would have a lower cost in relation to the diagnostic possibilities expressed.

It should be stressed that this study has some limitations due to the small size of the sample, the absence of a control sample and, obviously, the absence of a neuropathological confirmation of the diagnosis. However, we believe that further studies should be performed in virtue of the evidence to identify an AD MRI reproducible marker.

\section{Acknowledgements}

The study was supported by a grant of Fondazione Cassa di Risparmio di Roma and of Consorzio di bioingegneria e Informatica Medica (CBIM) of Pavia.

The authors want to thank Zsigmond Tamás Kincses, Nikoletta Szabó e László Vécsei from the Neuroimaging Section of the Neurológiai Clinic of the Albert Szent-Györgyi Medical and Pharmaceutical Center, Faculty 
of General Medicine of the University of Szeged, for their technical support and suggestions in analysis of diffusion MRI data, and Mauro Di Blasio for his technical support.

\section{Competing Interests}

The authors declare that they have no competing interests.

\section{Authors' Contributions}

EG have made substantial contributions to conception and design of the study, analysis and interpretation of data and drafted the manuscript.

RV have made substantial contributions to analysis and interpretation of data and drafted the manuscript.

FRP have made substantial contributions to analysis and interpretation of data and drafted the manuscript.

GB have made substantial contributions on analysis of data;

SG have made substantial contributions on analysis of data;

GL have made substantial contributions on analysis of MRS;

CC have made substantial contributions on analysis of data;

OP have made substantial contributions on statistical analysis of data;

SB have made substantial contributions to conception and design of the study and have been involved in revising the manuscript critically for intellectual content

All authors read and approved the final manuscript.

\section{References}

[1] McKhann, G., Drachman, D., Folstein, M., et al. (1984) Clinical Diagnosis of Alzheimer's Disease: Report of the NINCDS-ADRDA Work Group under the Auspices of Department of Health and Human Services Task Force on Alzheimer's Disease. Neurology, 34, 939-944. http://dx.doi.org/10.1212/WNL.34.7.939

[2] Dubois, B., Feldman, H.H., Jacova, C., et al. (2007) Research Criteria for the Diagnosis of Alzheimer’s Disease: Revising the NINCDS-ADRDA Criteria. The Lancet Neurology, 6, 734-746. http://dx.doi.org/10.1016/S1474-4422(07)70178-3

[3] Dubois, B., Feldman, H.H., Jacova, C., et al. (2010) Revising the Definition of Alzheimer' Disease: A New Lexicon. The Lancet Neurology, 9, 1118-1127. http://dx.doi.org/10.1016/S1474-4422(10)70223-4

[4] Frisoni, G.B., Winblad, B. and O’Brien, J.T. (2011) Revised NIA-AA Criteria for the Diagnosis of Alzheimer's Disease: A Step Forward but Not Yet Ready for Widespread Clinical Use. International Psychogeriatrics, 23, 1191-1196. http://dx.doi.org/10.1017/S1041610211001220

[5] Ghidoni, R., Benussi, L., Glionna, M., et al. (2008) Low Plasma Progranulin Levels Predict Progranulin Mutations in Frontotemporal Lobar Degeneration. Neurology, 71, 1235-1239. http://dx.doi.org/10.1212/01.wnl.0000325058.10218.fC

[6] Jack Jr., C.R., Petersen, R.C., O’Brien, P.C., et al. (1992) MR based hippocampal volumetry in the diagnosis of Alzheimer's disease. Neurology, 42, 183-188. http://dx.doi.org/10.1212/WNL.42.1.183

[7] Frisoni, G.B., Bianchetti, A., Geroldi, C. and Trabucchi, M. (1994) Measures of Medial Temporal Lobe Atrophy in Alzheimer's Disease. Journal of Neurology, Neurosurgery \& Psychiatry, 57, 1438-1439. http://dx.doi.org/10.1136/jnnp.57.11.1438

[8] Jack, C.R., Petersen, R.C., Xu, Y., et al. (1997) Medial Temporal Atrophy on MRI in Normal Aging and Very Mild Alzheimer's Disease. Neurology, 49, 786-794. http://dx.doi.org/10.1212/WNL.49.3.786

[9] Bobinski, M., deLeon, M.J., Convit, A., et al. (1999) MRI of Entorhinal Cortex in Alzheimer's Disease. The Lancet, 353, 38-40. http://dx.doi.org/10.1016/S0140-6736(05)74869-8

[10] Chételat, G., Landeau, B., Eustache, F., et al. (2005) Using Voxel-Based Morphometry to Map the Structural Changes Associated with Rapid Conversion in MCI: A Longitudinal MRI Study. NeuroImage, 27, 934-946. http://dx.doi.org/10.1016/j.neuroimage.2005.05.015

[11] Whitwell, J.L., Przybelski, S.A., Weigand, S.D., et al. (2007) 3D Maps from Multiple MRI Illustrate Changing Atrophy Patterns as Subjects Progress from Mild Cognitive Impairment to Alzheimer’s Disease. Brain, 130, 1777-1786.

[12] Fennema-Notestine, C., Hagler Jr., D.J., McEvoy, L.K., Fleisher, A.S., Wu, E.H., Karow, D.S. and Dale, A.M. (2009) Structural MRI Biomarkers for Preclinical and Mild Alzheimer’s Disease. Human Brain Mapping, 30, 3238-3253. http://dx.doi.org/10.1002/hbm.20744 
[13] Devanand, D.P., Bansal, R., Liu, J., Hao, X.J., et al. (2012) MRI Hippocampal and Entorhinal Cortex Mapping in Predicting Conversion to Alzheimer's Disease. Neuroimage, 60, 1622-1629. http://dx.doi.org/10.1016/j.neuroimage.2012.01.075

[14] Clerx, L., Jacobs, H.I., Burgmans, S., et al. (2013) Sensitivity of Different MRI-Techniques to Assess Gray Matter Atrophy Patterns in Alzheimer's Disease Is Region-Specific. Current Alzheimer Research, 10, 940-951. http://dx.doi.org/10.2174/15672050113109990158

[15] Bitsch, A., Bruhn, H., Vougioukas, V., et al. (1999) Inflammatory CNS Demyelination: Histopathologic Correlation with in Vivo Quantitative Proton MR Spectroscopy. AJNR American Journal of Neuroradiology, 20, 1619-1627.

[16] Jessen, F., Block, W., Traber, F., et al. (2000) Proton MR Spectroscopy Detects a Relative Decrease of N-Acetylaspartate in the Medial Temporal Lobe of Patients with AD. Neurology, 55, 684-688. http://dx.doi.org/10.1212/WNL.55.5.684

[17] Schuff, N., Capizzano, A.A., Du, A.T., et al. (2002) Selective Reduction of N-Acetylaspartate in Medial Temporal and Parietal Lobes in AD. Neurology, 58, 928-935. http://dx.doi.org/10.1212/WNL.58.6.928

[18] Watanabe, T., Shiino, A. and Akiguchi, I. (2010) Absolute Quantification in Proton Magnetic Resonance Spectroscopy Is Useful to Differentiate Amnesic Mild Cognitive Impairment from Alzheimer's Disease and Healthy Aging. Dementia and Geriatric Cognitive Disorders, 30, 71-77. http://dx.doi.org/10.1159/000318750

[19] Tumati, S., Martens, S. and Aleman, A. (2013) Magnetic Resonance Spectroscopy in Mild Cognitive Impairment: Systematic Review and Meta-Analysis. Neuroscience \& Biobehavioral Reviews, 37, 2571-2586. http://dx.doi.org/10.1016/j.neubiorev.2013.08.004

[20] Giugni, E., Vadalà, R., De Vincentiis, C., et al. (2010) The Brain’s Default Mode Network: A Mind "Sentinel” Role? Functional Neurology, 25, 189-190.

[21] Chhatwal, J.P. and Sperling, R.A. (2012) Functional MRI of Mnemonic Networks across the Spectrum of Normal Aging, Mild Cognitive Impairment, and Alzheimer's Disease. Journal of Alzheimer's Disease, 31, S155-S167.

[22] Weiler, M., Fukuda, A., Massabki, L.H., et al. (2014) Default Mode, Executive Function, and Language Functional Connectivity Networks Are Compromised in Mild Alzheimer’s Disease. Current Alzheimer Research, 11, $274-282$. http://dx.doi.org/10.2174/1567205011666140131114716

[23] Davatzikos, C., Bhatt, P., Shaw, L.M., et al. (2011) Prediction of MCI to AD Conversion, via MRI, CSF Biomarkers, and Pattern Classification. Neurobiology of Aging, 32, 2322.

[24] Jenkinson, M. and Smith, S. (2001) A Global Optimisation Method for Robust Affine Registration of Brain Images. Medical Image Analysis, 5, 143-156. http://dx.doi.org/10.1016/S1361-8415(01)00036-6

[25] Smith, S.M., Jenkinson, M., Woolrich, M.W., Beckmann, C.F., Behrens, T.E., Johansen-Berg, H., Bannister, P.R., De Luca, M., Drobnjak, I., Flitney, D.E., Niazy, R.K., Saunders, J., Vickers, J., Zhang, Y.Y., De Stefano, N., Brady, J.M. and Matthews, P.M. (2004) Advances in Functional and Structural MR Image Analysis and Implementation as FSL. Neuroimage, 23, S208-S219. http://dx.doi.org/10.1016/j.neuroimage.2004.07.051

[26] Smith, S.M., Jenkinson, M., Johansen-Berg, H., Rueckert, D., Nichols, T.E., Mackay, C.E., Watkins, K.E., Ciccarelli, O., Cader, M.Z., Matthews, P.M. and Behrens, T.E. (2006) Tract-Based Spatial Statistics: Voxelwise Analysis of Multi-Subject Diffusion Data. Neuroimage, 31, 1487-1505. http://dx.doi.org/10.1016/j.neuroimage.2006.02.024

[27] Andersson, J.L.R., Jenkinson, M. and Smith, S. (2007) Non-Linear Optimisation. FMRIB Technical Report.

[28] Rueckert, D., Sonoda, L.I., Hayes, C., Hill, D.L., Leach, M.O. and Hawkes, D.J. (1999) Nonrigid Registration Using Free-Form Deformations: Application to Breast MR Images. IEEE Transactions on Medical Imaging, 18, 712-721. http://dx.doi.org/10.1109/42.796284

[29] Nichols, T.E. and Holmes, A.P. (2002) Nonparametric Permutation Tests for Functional Neuroimaging: A Primer with Examples. Human Brain Mapping, 15, 1-25. http://dx.doi.org/10.1002/hbm.1058

[30] Behrens, T.E., Berg, H.J., Jbabdi, S., Rushworth, M.F. and Woolrich, M.W. (2007) Probabilistic Diffusion Tractography with Multiple Fibre Orientations: What Can We Gain? Neuroimage, 34, 144-155. http://dx.doi.org/10.1016/j.neuroimage.2006.09.018

[31] Dale, A.M., Fischl, B. and Sereno, M.I. (1999) Cortical Surface-Based Analysis: I. Segmentation and Surface Reconstruction. Neuroimage, 9, 179-194. http://dx.doi.org/10.1006/nimg.1998.0395 
Scientific Research Publishing (SCIRP) is one of the largest Open Access journal publishers. It is currently publishing more than 200 open access, online, peer-reviewed journals covering a wide range of academic disciplines. SCIRP serves the worldwide academic communities and contributes to the progress and application of science with its publication.

Other selected journals from SCIRP are listed as below. Submit your manuscript to us via either submit@scirp.org or Online Submission Portal.
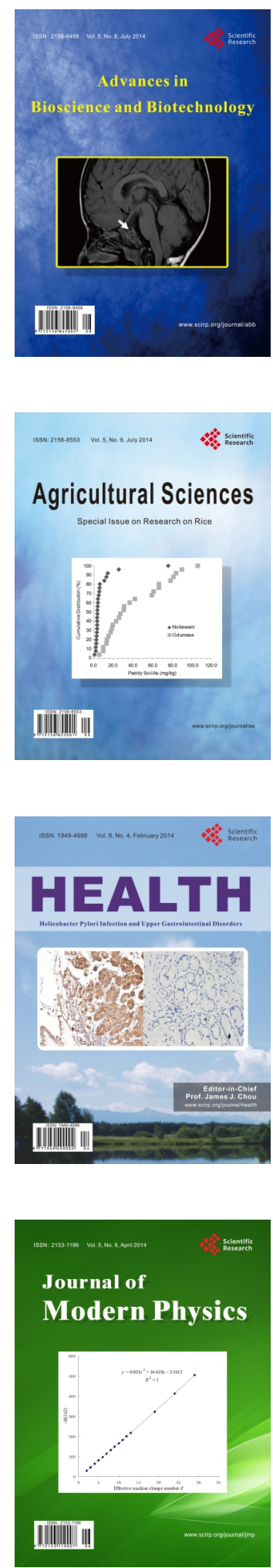
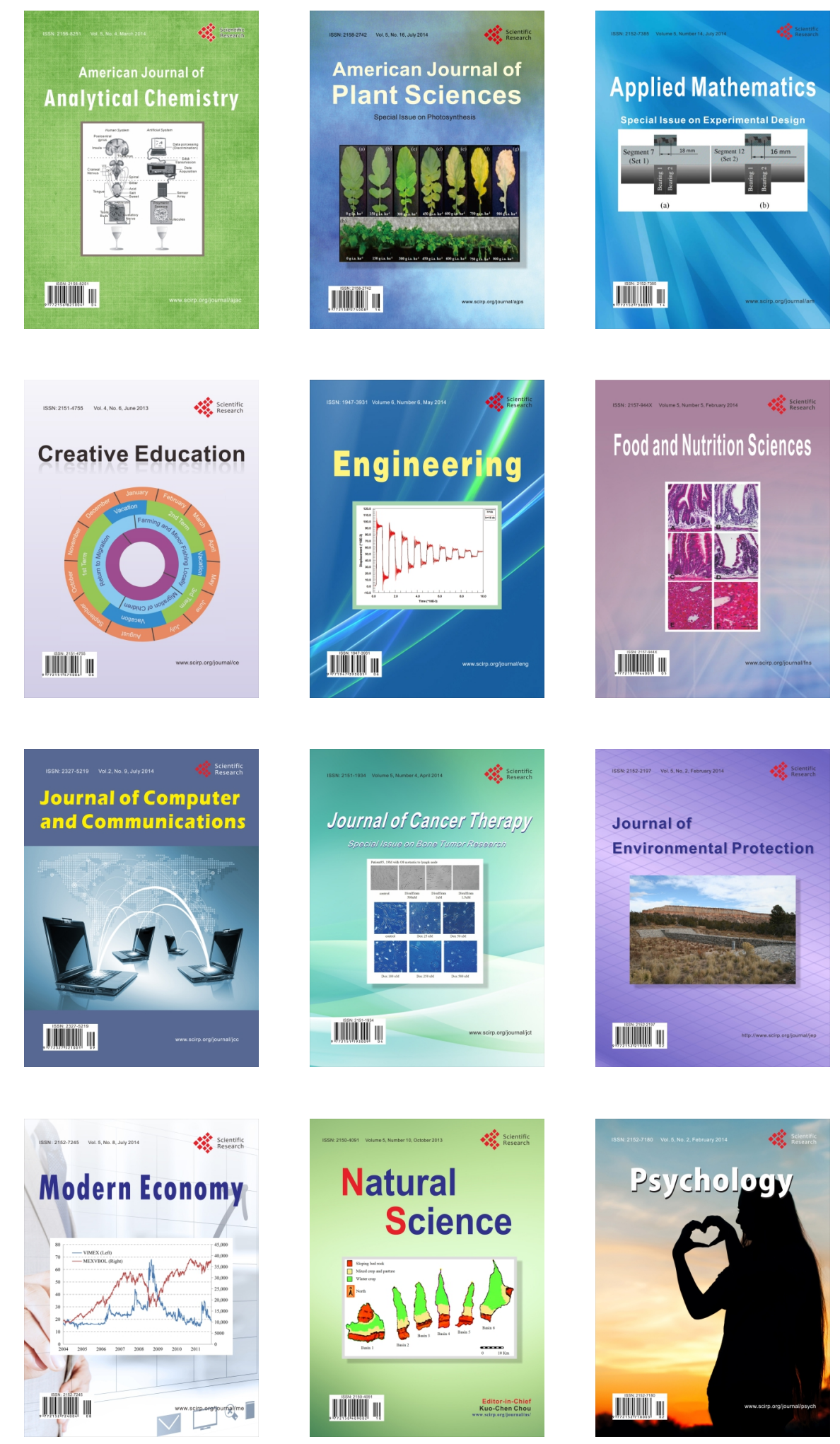